\title{
Serum 25-Hydroxyvitamin D in Patients with Juvenile Idiopathic Arthritis Mohamed Almalky ${ }^{1}$, Mervat Hesham ${ }^{1}$, Ayman Marei ${ }^{2}$, Mogeep El-Rahman Ibrahim*1 \\ Departments of ${ }^{1}$ Pediatrics \& ${ }^{2}$ Microbiology and Immunology, Faculty of Medicine, Zagazig University. *Corresponding author: Mogeep El-Rahman Dyaa Ahmed Mostafa Ibrahim, Mobile: +20)01033808665, \\ Email: mogeepdyaa@gmail.com
}

\begin{abstract}
Background: Vitamin D has been implicated in the pathogenesis of a lot of autoimmune diseases. While the roles of vitamin $\mathrm{D}$ in rheumatic arthritis in adult have been investigated, less is known about the role of vitamin $\mathrm{D}$ in juvenile idiopathic arthritis (JIA).

Objectives: Evaluation of the level of serum $25(\mathrm{OH})$ vitamin $\mathrm{D}$ in patients with juvenile idiopathic arthritis and its relationship to the disease activity.

Methods: This was a case-control study included 36 children who were divided into two equal groups of matched age and sex. The first group (case group) consisted of 18 patients with JIA. The second group (control group) consisted of 18 healthy children. $25(\mathrm{OH})$ vitamin D level is measured in both groups and its relation to disease manifestations and disease activity using JADAS 27 score was determined.

Results: The mean value of vitamin D serum level was significantly lower in patients than in healthy control. There was a negative correlation between vitamin $\mathrm{D}$ level and both disease activity and number of joints affected among the studied patients.

Conclusion: Serum vitamin D levels were reduced in children with JIA. In addition, there was a significant negative correlation between serum vitamin D levels and both the number of affected joints and the disease activity as measured by JADAS 27 score.
\end{abstract}

Keywords: Juvenile idiopathic arthritis, vitamin D, 25 hydroxy vitamin D, JADAS 27 score.

\section{INTRODUCTION}

Vitamin D is a steroid hormone synthesized in the skin through sunlight exposure or ingested in the diet and its active form is 1,25 dihydroxy vitamin $\mathrm{D}$ $\left(1,25[\mathrm{OH}]_{2} \mathrm{D}\right)^{(\mathbf{1})}$. It is not only a regulator of calcium and phosphate homeostasis, but also has numerous extra-skeletal effects including the significant impact on the cardiovascular system, central nervous system, endocrinal system and immune system as well as on cell differentiation and cell growth ${ }^{(2)}$. As JIA (the most common chronic rheumatic disease in childhood) is an autoimmune disease, deficiency of vitamin D may play a role in its pathogenesis through its immune effect. The mechanisms by which vitamin $\mathrm{D}$ affects the pathogenesis and severity of JIA are multiple and include modulation of transcription pathways leading to cytokine production, regulation of the expression of cyclooxygenase 2 and lipoxygenase genes, and it may directly interferes with cytokine gene expression and signaling, favoring a change from a T helper-1 (Th-1) into a Th-2 cytokine profile, as well as a decrease in Th-17 response ${ }^{(3)}$.

So, suppression of autoimmune diseases generally and JIA specifically involves the vitamin D hormone interaction with $\mathrm{T}$ helper lymphocytes, which in turn suppress the inflammatory responses of $\mathrm{T}$ helper-1 lymphocytes ${ }^{(4)}$.

There is a controversy regarding the level of vitamin $\mathrm{D}$ that influences both the prevalence and the outcome of JIA 8 . Since there is no clearly defined normal levels for vitamin D in children, cutoff levels proposed for adults have been used in studies with children making it difficult to do an accurate assessment of its level in children with JIA and to assess its relation to disease activity and outcome ${ }^{(5)}$.

The aims of this study were to evaluate vitamin D status in patients of juvenile idiopathic arthritis (JIA) compared to normal children of matched age and sex (case control study), and also to examine whether there is an association between serum level of 25hydroxyvitamin $\mathrm{D}[25(\mathrm{OH}) \mathrm{D}]$ and disease activity in JIA.

\section{SUBJECTS AND METHODS}

\section{Technical design:}

- Site of study: This is case-control study that was done in Pediatric Department, Zagazig University Hospitals.

- Sample size: In this study 36 children were included, divided into two equal groups of matched age and sex. The first group (case group) consisted of 18 patients with Juvenile idiopathic arthritis, while the second group (control group) consisted of 18 healthy children.

The number of cases was estimated according to the prevalence of JIA in Sharkia governorate and the number of cases of JIA regularly followed up in Department of Pediatrics, Zagazig University Hospital ${ }^{(6)}$.

\section{- Inclusion criteria:}

This article is an open access article distributed under the terms and conditions of the Creative Commons Attribution (CC BY-SA) license (http://creativecommons.org/licenses/by/4.0/) 
1. Children diagnosed with juvenile idiopathic arthritis with both active and inactive disease status were enrolled.

2. Both sexes.

\section{- Exclusion criteria:}

1. Known rickets patients

2. Patients who were receiving treatment (except corticosteroids) that could influence vitamin D status like anticonvulsant.

3. Patients taking vitamin D supplementation.

\section{Ethical approval:}

- Approval of the study was obtained from the Institutional Review Board - Zagazig University (IRB-ZU). An informed consent was taken from the patients and their parents.

3. Operational Design:

a) History:

- Collected data included:

1. Personal data: age, sex.

2. Disease duration.

b) Medication history of corticosteroid, non-steroidal anti-inflammatory drugs (NSAIDs), methotrexate, sulfasalazine and biological drugs.

c) All demographic data, clinical manifestations, laboratory values, disease activity indices, and medications were reviewed and calculated from previous medical records in Pediatrics Hospital, Zagazig University.

d) Examination:

\section{General examination:}

- Vital signs.

- Anthropometric measurements.

2. Systemic examination:

- Chest.

- Heart.

- Abdomen.

- Musculoskeletal examination.

3. Specific examination \& classification:

- Symptoms of juvenile rheumatoid arthritis in order to classify the disease subtype.

- Classification \& diagnosis of patients according to criteria of the International League of Associations for Rheumatology (ILAR).

- Disease activity was evaluated using a previously validated score, JADAS-27 ${ }^{(7)}$.

\section{e) Radiological study:}

$\mathrm{X}$-ray were performed just in clinically active joints, with disease duration more than 6 months, in order to evaluate the radiographic damage (erosions or joint space narrowing).

f) Laboratory tests:

1) Complete blood count (CBC): by Cell-Dyne 1700 cell counter; as we consider leukopenia (WBCs <4000/mm3), thrombocytopenia (platelets $<100,000 / \mathrm{mm} 3)$, anemia $(\mathrm{Hb}<9 \mathrm{~g})$.
2) Erythrocyte sedimentation rate (ESR): by sodium citrate.

3) Rheumatoid factor: by rheumatoid factor IgM ELISA.

4) Antinuclear antibody (ANA): by Indirect Immunofluorescence.

5) Serum 25-OH D concentrations: by Euro immune ELISA ${ }^{(8)}$.

\section{Statistical analysis:}

The collected data were coded, tabulated, and statistically analyzed using IBM SPSS statistics (Statistical Package for Social Sciences) software version 21.0 IBM Corp., Chicago, USA, 2013. Descriptive statistics were done for quantitative data as minimum \& maximum of the range as well as mean \pm SD (standard deviation) for quantitative parametric data, while it was done for qualitative data as number and percentage. Inferential analyses for independent variables were done using Chi square test for differences between proportions and student t-test for continuous variables. For more than two group ANOVA test was used. Person correlation coefficient was used to find the correlation between each two variables. The level of significance was taken at $\mathrm{P}$ value $\leq 0.05$ otherwise was non-significant.

\section{RESULTS}

Thirty-six children were included in our study, eighteen patients of JIA (cases group) and eighteen healthy children (control group). The age of patients ranged from 2 to 13 years with mean age $6.81 \pm 3.15$. Nine patients were females $(50 \%)$ and nine patients were males $(50 \%)$, which was nearly similar to control group whose ages ranged from 3 to 10 years with mean age $6.06 \pm 2.18$, ten control children were females $(55.6 \%)$ and eight were males $(44.4 \%)$.

There were nine patients with polyarticular JIA, (50\%), five patients with systemic onset JIA (27.2\%) and four patients with oligoarticular JIA (22.8\%) no other types of JIA had been found. All of our patients $(100 \%)$ had fever, joint swelling, joint tenderness and limitation of movement while only five had skin rash $(27.2 \%)$ and three patients had enlarged lymph nodes $(16.7 \%)$ and no other disease-related manifestations had been detected. The number of joint affected ranged from 2 to 20 with median of 10 joints affected. All of our patients had high disease activity by JADAS 27 score (table 1). It took one to six months to diagnose our cases which was a relatively long time. All our patients received non-steroidal anti-inflammatory drugs, corticosteroids and methotrexate while half of them received hydroxychloroquine and none of them received other therapies like biological therapy. Only one patient had Rheumatoid factor and only one patient had raised ANA level.

Vitamin D was significantly lower in cases than control regarding both level (table 3 ) and status (table 
4) while there was no significant difference between different types of JIA regarding vitamin D status (table 2). The duration of treatment was significantly less in patients with higher levels of vitamin D (table 5).

The response to treatment was better in patients with higher levels of vitamin D (table 6).
There was negative correlation between level of vitamin $\mathrm{D}$ and disease activity by JADAS score (table 5) and (figure 1).

Table (1): Juvenile arthritis disease activity score (JADAS 27 score) among the cases group

\begin{tabular}{|l|l|c|}
\hline & \multicolumn{1}{|c|}{ JADAS 27 score } & Value \\
\hline & Mean \pm SD & $29.89 \pm 10.09$ \\
& Median & 31 \\
& Range & $10-44$ \\
\hline Activity & High & $18(100)$ \\
& Low & $0(0)$ \\
\hline
\end{tabular}

The mean JADAS 27 score among the studied patients was 29.89 ranging from 10 to 44 .

All of them had highly active disease, based on cutoff $\geq 8.5$ for polyarticular JA and $\geq 4.2$ for oligoarticular (table 1 ).

Table (2): Relation between vitamin D status and type of disease

\begin{tabular}{|c|c|c|c|c|c|}
\hline $\begin{array}{c}\text { JIA type } \\
\text { Vitamin D status }\end{array}$ & $\begin{array}{c}\text { Polyarticular type } \\
\text { N=9 }(\%)\end{array}$ & $\begin{array}{c}\text { Oligoarticular type } \\
\mathrm{N}=4(\%)\end{array}$ & $\begin{array}{c}\text { Systemic type } \\
\mathrm{N}=5(\%)\end{array}$ & $X^{2}$ & $\mathbf{P}$ \\
\hline Sufficient & $0(0 \%)$ & $2(50 \%)$ & $1(20 \%)$ & \multirow{3}{*}{2.583} & \multirow{3}{*}{0.46} \\
\hline Insufficient & $1(22.2 \%)$ & $1(25 \%)$ & $2(40 \%)$ & & \\
\hline Deficient & $8(77.8 \%)$ & $1(25 \%)$ & $2(40 \%)$ & & \\
\hline
\end{tabular}

Table (3): Comparison between the studied groups regarding vitamin D level

\begin{tabular}{|l|c|c|c|c|}
\hline & Cases group & Control group & & \multirow{2}{*}{$\mathbf{P}$} \\
\cline { 2 - 5 } & Mean \pm SD & Mean \pm SD & Z & $<0.001^{*}$ \\
\hline Vitamin D level $(\mathbf{n g} / \mathbf{m l})$ & $19.8 \pm 3.02$ & $29.6 \pm 4.4$ & $\mathrm{t}(-5.559)$ & \\
\hline
\end{tabular}

Table (4): Comparison between the studied groups regarding vitamin D status

\begin{tabular}{|c|c|c|c|c|}
\hline \multirow[t]{2}{*}{ Vitamin D status } & Cases group & Control group & \multirow[t]{2}{*}{$X^{2}$} & \multirow[t]{2}{*}{$\mathbf{P}$} \\
\hline & $\mathrm{N}=18(\%)$ & $\mathrm{N}=18(\%)$ & & \\
\hline Sufficient & $3(16.7)$ & $12(66.7)$ & \multirow{3}{*}{13.844} & \multirow{3}{*}{$0.001 * *$} \\
\hline Insufficient & $4(22.2)$ & $5(27.8)$ & & \\
\hline Deficient & $11(61.1)$ & $1(5.6)$ & & \\
\hline $\mathbf{O R}^{¥}$ & 26.71 & CI & \multicolumn{2}{|c|}{$2.88-248.03$} \\
\hline
\end{tabular}

Table (5): Correlation between vitamin D level and number of joint affected, juvenile arthritis disease activity score (JADAS 27 score) and duration of treatment

\begin{tabular}{|l|c|c|}
\hline \multirow{2}{*}{} & $\mathbf{2}$ & \multicolumn{2}{|c|}{ Vitamin D level } \\
\cline { 2 - 3 } & $\mathbf{R}$ & $\mathbf{P}$ \\
\hline JADAS 27 score & $\mathbf{- 0 . 9 3 9}$ & $<\mathbf{0 . 0 0 1 * *}$ \\
\hline Duration of treatment & $\mathbf{0 . 9 7 7}$ & $<\mathbf{0 . 0 0 1 * *}$ \\
\hline Number of joint affected & $\mathbf{- 0 . 7 9 6}$ & $<\mathbf{0 . 0 0 1 * *}$ \\
\hline
\end{tabular}




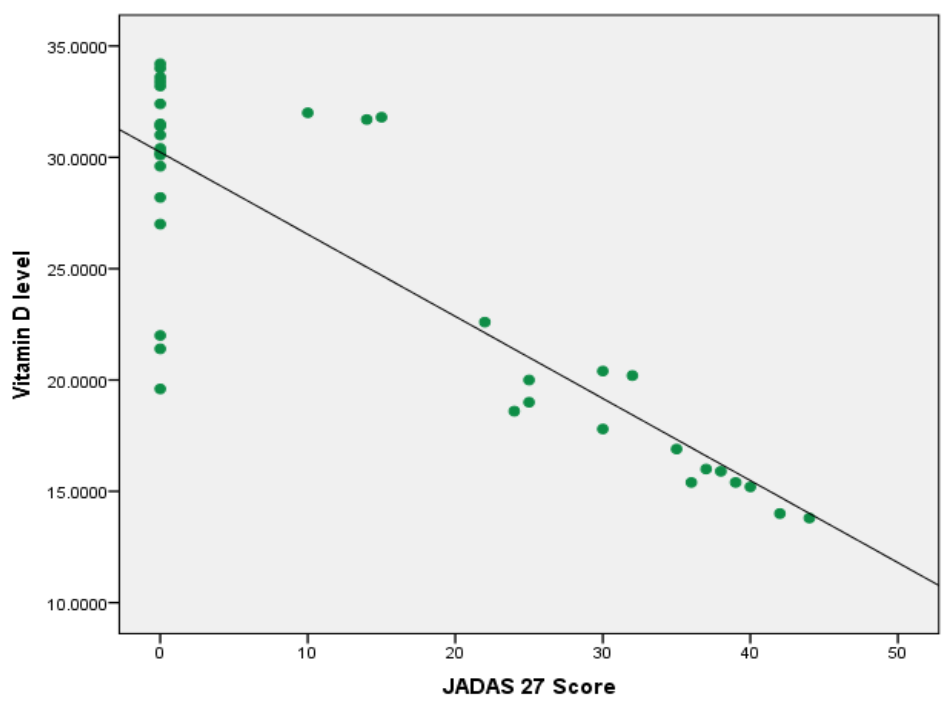

Figure (1): Scatter dot graph showing Correlation between vitamin D level and JADAS 27 score.

Table (6): Relation between response to treatment and vitamin D status

\begin{tabular}{|l|c|c|c|c|c|}
\hline \multirow{2}{*}{} & \multicolumn{3}{|c|}{ Vitamin D status } & \multirow{2}{*}{$X^{2}$} & \multirow{2}{*}{$\mathrm{P}$} \\
\cline { 2 - 4 } & Sufficient & Insufficient & Deficient & & \\
\cline { 2 - 5 } & $\mathrm{N}=3(\%)$ & $\mathrm{N}=4(\%)$ & $\mathrm{N}=11(\%)$ & & \\
\hline Symptom decrease: & $0(0)$ & $0(0)$ & $7(63.6)$ & & \\
No & $3(100)$ & $4(100)$ & $4(36.4)$ & 7.289 & $0.026^{*}$ \\
\hline
\end{tabular}

\section{DISCUSSION}

In our study polyarticular JIA was the commonest type among studied patients $(50 \%)$, which is similar to the studies of El Deriny and Mannaa ${ }^{(9)}$ and De

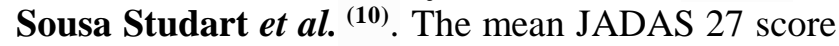
among the studied patients was $29.89 \pm 10.09$ that ranged from 10 to 44 . All of our patients had highly active disease based on cutoff $\geq 8.5$ for polyarticular JA and $\geq 4.2$ for oligoarticular. This is opposed by De Sousa Studart et al. ${ }^{\left({ }^{10}\right)}$ as the mean JADAS-27 score was $9.1 \pm 10.7$ and only $44 \%$ of patients were considered highly active. This may be explained by that most of our patients were recently diagnosed and only patients with highly active disease are regularly followed up in outpatient clinic.

In current study vitamin $\mathrm{D}$ levels were lower in cases than control as in cases it ranged from 13.8-32 $\mathrm{ng} / \mathrm{ml}$ with a mean of $19.82 \pm 6.02$ while in control group ranged from $19.6-34.2 \mathrm{ng} / \mathrm{ml}$ with a mean of $29.62 \pm 4.44$. These results agree with the study of EI Deriny and Mannaa ${ }^{(9)}$ (ranged from 8.9-28 ng/ml with a mean of $16.1 \pm 6.1$ in case group and ranged from $9-42 \mathrm{ng} / \mathrm{ml}$ with a mean of $20.6 \pm 6.2$ in control group) and with Dağdeviren-Çakır et al. ${ }^{(11)}$ (mean was $18.9 \pm 11$ in cases group and $26.71 \pm 10.54$ in control). Also, with Stagi et al. ${ }^{(12)}$ (mean was $21.8 \pm$ 8.2 in cases group and $29.8 \pm 11.2$ in control). Most of our patients $(61.1 \%)$ had deficient vitamin D versus $5.6 \%$ of the control group while two thirds $(66.7 \%)$ of control had sufficient vitamin D versus $16.7 \%$ of patients. Meanwhile, there was negative correlation between vitamin $\mathrm{D}$ level and the number of joints affected among the studied patients.

This is in contrast to the study of De Sousa Studart et al. ${ }^{(10)}$ who revealed that there was no significant difference in vitamin $\mathrm{D}$ level between cases and control. This difference may be because his study was done in low altitude area and this finding was confirmed in a review article done by Finch $\boldsymbol{e t}$ al. (13).

Our study revealed that vitamin D was more deficient in patients with higher disease activity detected by JADAS 27 scoring system. This is consistent with the finding of Stagi et al. ${ }^{(12)}$, Çomak et al. ${ }^{(14)}$, Bouaddi et al. ${ }^{(15)}$ and Sengle et al. ${ }^{(16)}$. This match with most of the published data comparing vitamin D with JIA disease activity even those who used different scoring system like Childhood Health Assessment Questionnaire (CHAQ) and American College of Rheumatology Pediatric 30 Criteria (ARC Peds 30). Only few studies showed high vitamin D level during disease activity like that done by Miettinen et al. ${ }^{(17)}$, but many studies revealed no relation between disease activity and vitamin D level like that done by Dağdeviren-çakır et al. ${ }^{(11)}$ and Munekata et al. (18). This variability could be explained by that there is no universal cutoff value to determine level of vitamin D deficiency or even insufficiency making it too difficult to unify these data to date. There are many confounding variables (sun exposure, season, and vitamin D intake, altitude) that we need to control. So, many authors still believe that 
the evidence to support a relationship between vitamin $\mathrm{D}$ and disease activity with autoimmune diseases in humans is correlative and not causative. Long-term, adequately powered randomized studies, which control for confinding variables (sun exposure, season, and vitamin $\mathrm{D}$ intake) that are required to confirm a causative relationship between vitamin D and disease activity ${ }^{(5,19)}$.

Our study stated that patients with higher levels of vitamin $\mathrm{D}$ are more responsive to treatment and this finding matches with that of Kim et al. ${ }^{(20)}$ and Stark et al. (22) who recommended vitamin D supplementation for patients with rheumatoid arthritis for the prevention and treatment of osteoporosis as well as for its possible inhibitory effect on disease activity. In contrast, Tang et al. ${ }^{(22)}$ revealed that cholecalciferol supplementation (2000 IU/day) for 24 weeks raised serum levels of $25 \mathrm{OH}$ D in JIA patients but did not reduce disease activity or improve bone mineral density (BMD).

\section{CONCLUSION}

In conclusion serum vitamin $\mathrm{D}$ levels were reduced in children with JIA. In addition, a significant negative correlation between serum vitamin $\mathrm{D}$ levels and both the number of affected joints and the disease activity measured by JADAS 27 score were found.

Conflict of interest: The authors declare no conflict of interest.

Funding sources: The authors had no funding to report.

\section{Acknowledgement:}

The authors are grateful for the patients without whom this study would not have been done.

\section{REFERENCES}

1. Gröber U, Holick MF (2013): Vitamin D. Die Heilkraft des Sonnenvitamins. 2.Auflage, 304 S., Wissenschaftliche Verlagsgesellschaft, https://www.amazon.de/Vitamin-D-Die-HeilkraftSonnenvitamins/dp/3804732763

2. Wacker M, Holick MF (2013): Vitamin D - effects on skeletal and extraskeletal health and the need for supplementation. Nutrients, 5: 111-148.

3. Wöbke TK, Sorg BL, Steinhilber D (2014): Vitamin D in inflammatory diseases. Front Physiol., 5: 244-248.

4. Griffin MD, Lutz W, Phan VA et al. (2001): Dendritic cell modulation by 1 alpha, 25 dihydroxy vitamin D3 and its analogs: a vitamin $\mathrm{D}$ receptor-dependent pathway that promotes a persistent state of immaturity in vitro and in vivo. Proc Natl Acad Sci USA., 98 (12): 6800-6805.

5. Nisar MK, Masood F, Cookson P et al. (2013): What Do We Know About Juvenile Idiopathic Arthritis and Vitamin D? A Systematic Literature Review and Meta-Analysis of Current Evidence. Clin Rheumatol., 32 (6): 729-34.

6. El-Najjar AR, Nejm MG, El-Sayed WM (2014): The relationship between depression, disease activity and physical function in Juvenile Idiopathic Arthritis patients in Zagazig University Hospital - Egypt. The Egyptian Rheumatologist, 36: 145-150.

7. Consolaro A, Giancane G, Schiappapietra B et al. (2016): Clinical outcome measures in juvenile idiopathic arthritis. Pediatr Rheumatol Online J., 14: 23.

8. Holick MF, Biancuzzo RM, Chen TC et al. (2008): Vitamin D2 is as effective as vitamin D3 in maintaining circulating concentrations of 25-hydroxyvitamin D. J Clin Endocrinol Metab., 93: 677-681

9. El Deriny G, Mannaa H (2019): Vitamin D status in children with juvenile idiopathic arthritis in Alexandria University Children's Hospital. European Journal of Pharmaceutical and Medical Research, 6 (3): 341-350.

10. De Sousa SA, Leite AC, Marinho AL et al. (2015): Vitamin D levels in juvenile idiopathic arthritis from an equatorial region. Rheumatology International, 35: 1717-1723.

11. Dağdeviren-Çakır A, Arvas A, Barut Ket al. (2016): Serum vitamin $\mathrm{D}$ levels during activation and remission periods of patients with juvenile idiopathic arthritis and familial Mediterranean fever. Turk J Pediatr., 58: 125-131.

12. Stagi S, Bertini F, Cavalli L et al. (2014): Determinants of vitamin D levels in children, adolescents, and young adults with juvenile idiopathic arthritis. The Journal of Rheumatology, 41 (9): 1884-1892.

13. Finch SL, Rosenberg AM, Vatanparast $H$ (2018): Vitamin D and juvenile idiopathic arthritis. Pediatric Rheumatology, 16: 34-38.

14. Çomak E, Doğan ÇS, Uslu-gökçeoğlu A et al. (2014): Association between vitamin D deficiency and disease activity in juvenile idiopathic arthritis. Turk J Pediatr., 56: 626-31.

15. Bouaddi I, Rostom S, El Badri D et al. (2014): Vitamin D concentrations and disease activity in Moroccan children with juvenile idiopathic arthritis. BMC Musculoskelet Disord., 15: 115-118.

16. Sengler C, Zink J, Klotsche J et al. (2018): Vitamin D deficiency is associated with higher disease activity and the risk for uveitis in juvenile idiopathic arthritis - data from a German inception cohort. Arthritis Research \& Therapy, 20 (1): 276-278.

17. Miettinen ME, Kinnunen L, Harjutsalo V et al. (2013): Serum 25-hydroxyvitamin D in juvenile idiopathic arthritis patients in Finland-letters to the editors. Clin Exp Rheumatol., 31: 988.

18. Munekata RV, Terreri MTRA, Peracchi OAB et al. (2013): Serum 25-hydroxyvitamin D and biochemical markers of bone metabolism in patients with juvenile idiopathic arthritis. Braz J Med Biol Res., 46: 98-102.

19. Cutolo M, Otsa K, Uprus $M$ et al. (2007): Vitamin $D$ in rheumatoid arthritis. Autoimmun Rev., 7: 59-64.

20. Kim TH, Choi SJ, Lee YH et al. (2012): Combined therapeutic application of mTOR inhibitor and vitamin D (3) for inflammatory bone destruction of rheumatoid arthritis. Med Hypotheses, 79 (6): 757-60.

21. Stark LJ, Davis AM, Janicke DM et al. (2006): A randomized clinical trial of dietary calcium to improve bone accretion in children with juvenile rheumatiod arthritis. Pediatrics, 148: 501-7.

22. Tang T, Zhang Y, Luo C et al. (2019): Adjunctive Vitamin $\mathrm{D}$ for the Treatment of Active Juvenile Idiopathic Arthritis An Open-Label, Prospective, Randomized Controlled Trial. Exp Ther Med., 18 (6): 4921-4926. 